\title{
COVID-19 in Neonates: A Call for Standardized Testing
}

\author{
Sindhu Sivanandan, ${ }^{1}$ Deepak Chawla, ${ }^{2}$ Praveen Kumar ${ }^{3}$ and Ashok K Deorari ${ }^{4}$ for the National Neona- \\ TOLOGY FORUM, INDIA \\ From Departments of Neonatology, ' Jawaharlal Institute of Postgraduate Medical Education and Research (JIPMER), \\ Puducherry; ${ }^{2}$ Government Medical College and Hospital, and Departments of Pediatrics, ${ }^{3}$ PGIMER, Chandigarh; and ${ }^{4}$ All India \\ Institute of Medical Sciences, New Delhi; India. \\ Correspondence to: Dr. Praveen Kumar, Professor and Head, Division of Neonatology Department of Pediatrics, PGIMER, \\ Chandigarh 160012, India.drpkumarpgi@gmail.com
}

\begin{abstract}
The limited evidence on neonatal coronavirus disease (COVID-19) suggests that vertical transmission of severe acute respiratory syndrome coronavirus 2 (SARS-CoV-2) is rare, and most neonates seem to acquire the infection postnatally through respiratory droplets and contact. Testing of neonates with perinatal or postnatal exposure to COVID-19 infection plays a vital role in the early diagnosis, management and institution of infection prevention measures thereby cutting off the chain of epidemic transmission. A recently concluded online neonatal COVID-19 conference conducted by the National Neonatology Forum (NNF) of India and a nationwide online survey pointed to substantial variation in neonatal testing strategies. We, herein, summarize the relevant literature about the incidence and outcomes of neonatal COVID-19 and call for a universal and uniform testing strategy for exposed neonates. We anticipate that the testing strategy put forth in this article will facilitate better management and safe infection prevention measures among all units offering neonatal care in the country.
\end{abstract}

Keywords: Nucleic acid testing, RT-PCR, Rapid antigen test, SARS-CoV-2.

Published Online: October 24, 2020; PII: S097475591600254

T The severe acute respiratory syndrome coronavirus 2 (SARS-CoV-2) pandemic has affected over 41 million people globally and has caused more than 1 million deaths [1]. Pediatric cases account for $2-8 \%$ of diagnosed coronavirus disease (COVID-19) [2], and three-quarters acquire the disease from an infected family member. While the disease is generally milder in children when compared to adults, a small proportion require hospitalization or intensive care, and there is an increasing recognition of a Multisystem inflammatory syndrome related to COVID-19 illness in children (MIS-C), a severe condition with potential long-term consequences $[3,4]$. The infection rate in this vulnerable group is increasing [5], and the reported burden is likely an underestimate due to a higher proportion of asymptomatic infections, and lack of standardized testing protocols. Amongst neonates, the risk of vertical transmission is rare and most cases are reported to be acquired horizontally from infected contacts [6]. However, the modes of transmission and the impact of COVID-19 among neonates is less well characterized.

The National Neonatology Forum (NNF) of India in collaboration with Federation of Obstetric and Gynaecological Societies of India (FOGSI), and Indian Academy of Pediatrics (IAP) has published evidence- based recommendations for perinatal-neonatal COVID19 [7]. In an online NNF COVID-19 conference held on 10 July, 2020, substantial variability in testing strategy for SARS-CoV-2 exposed neonates between centers was evident. Following this, NNF India conducted a crosssectional nationwide online survey in July-August, 2020 to investigate this variability further. The call for participation was made via email and social media. A total 45 hospitals responded till 20 August, 2020, of which 25 were COVID-designated hospitals. All hospitals tested neonates born to COVID-19 positive mothers once or at multiple time points; $9(20 \%)$ tested neonates at birth, $18(40 \%)$ by 24 hours, $16(36 \%)$ by 48 hours and $49 \%$ between days 5-7 (Fig. 1). While $44 \%$ did not do repeat testing, others repeated it after varying time periods irrespective of initial results. The majority $(97 \%)$ used reverse-transcriptase polymerase chain reaction (RT-PCR) test on oro-nasopharyngeal swab. Among neonates presenting to a health facility with symptoms, 11 $(25 \%)$ of the hospitals tested all such neonates while others selectively tested based on certain criteria like history of contact, respiratory symptoms or as screening prior to surgery.

Lack of standardized testing protocols has important implications for the management of the neonate, infection prevention and control practices, as well as for 


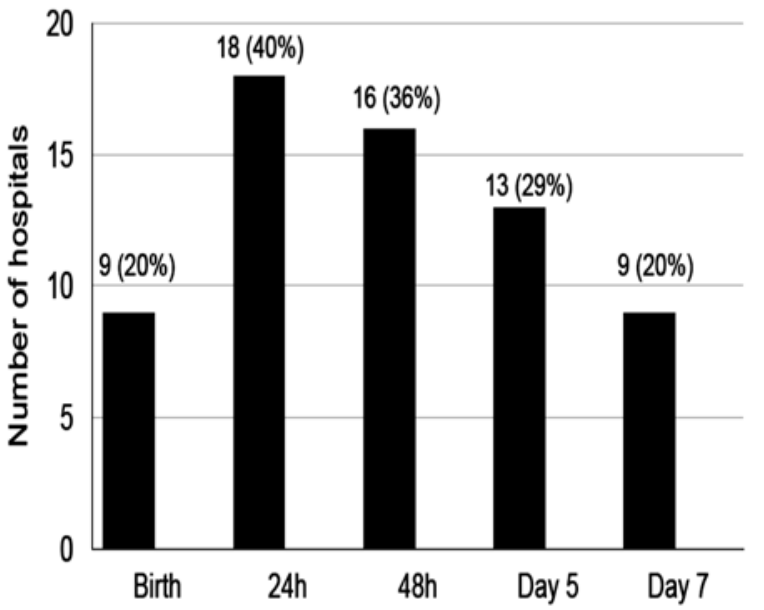

(a) Timing of first RT-PCR testing

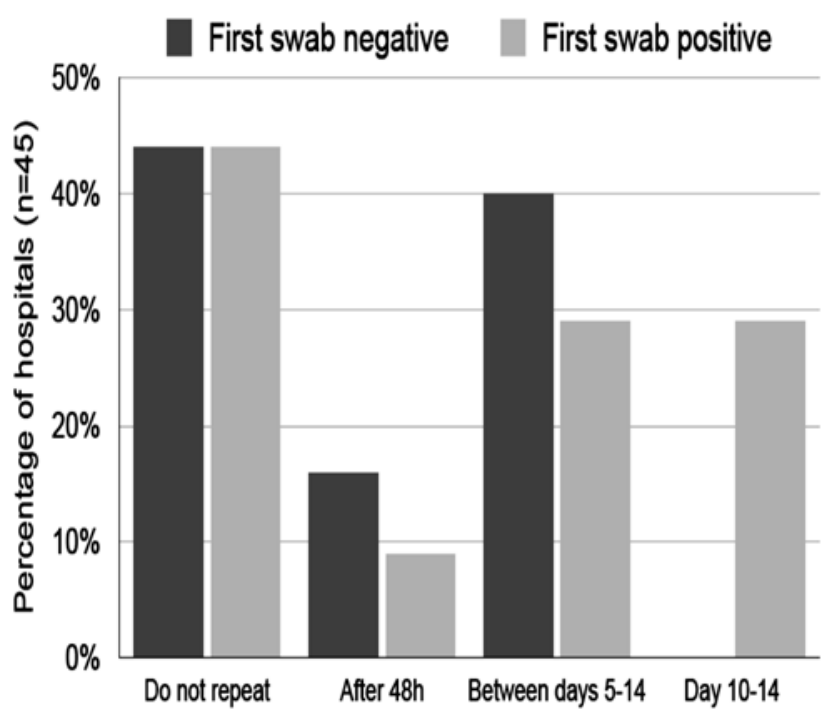

(b) Timing of repeat RT-PCR testing

Fig. 1 Testing strategies adopted by 45 participating hospitals managing neonates with perinatal or postnatal COVID-19 exposure Results of NNF cross-sectional online survey (July-August, 2020).

understanding disease epidemiology. In this article, we review the available evidence on neonatal SARS-CoV-2 infection and make a case for uniform and universal testing of COVID-19 exposed neonates.

\section{NEONATAL SARS-COV-2 INFECTION}

\section{Incidence}

Neonates, like infants and children have a lower incidence of SARS-CoV-2 infection. In a systematic review, Dhir, et al. [6] reviewed the outcomes of 1141 neonates born to COVID-19 positive women reported in 45 case series. Two-thirds were delivered by cesarean section, a quarter were preterm, and among 1005 (88\%) neonates tested, $39(3.9 \%)$ were found to be positive by RT-PCR. In the perinatal COVID-19 registry of the American Academy of Pediatrics, 2962 mother-infant dyads had been enrolled till 29 August 2020 from 264 centers across the United States of America (USA) [8]. In this registry, $2561(86 \%)$ neonates underwent testing by RT-PCR and 45 (1.8\%) tested positive for SARS-CoV-2. Two-thirds of the infants were delivered vaginally, and about a half were roomed-in with their mothers, and were breastfed directly or with expressed milk by mothers themselves. Of 26 neonatal deaths in this cohort, none were related to COVID-19. In a large series from Mumbai, India, only 3 out of 131 (2.2\%) neonates born to COVID-19 mothers tested positive within 24 hours of birth [9]. These neonates subsequently turned negative when retested on day 5 . In this series, $50 \%$ of the infants were delivered vaginally and rooming-in and breast- feeding were encouraged. Reports from other national databases have shown a variable risk of perinatal transmission; 2 (4.9\%) out of 41 tested from Kuwait [10], $12(5 \%)$ out of 240 tested from the UK Obstetric Surveillance System (UKOSS) [11], 9 (6.1\%) out of 147 tested from the Italian Obstetric Surveillance System (ItOSS) [12], 4 (3.3\%) of 120 tested from Turkey [13], and $1(2.7 \%)$ of 36 tested (2.7\%) from France [14].

\section{Mode of Transmission}

SARS-CoV-2 infection can pass from mother to fetus/ neonate through trans-placental route or during delivery from exposure to maternal blood or secretions. Postnatally, the infection can be transmitted from infected mother or caregivers through aerosols or direct contact. Initially, with limited information, there was no consensus on the type of samples (maternal and neonatal), and timing and type of testing to categorize if the COVID-19 infection was congenital, or acquired at birth or postnatally. Some experts have put forth classification schema, but none is universally accepted currently $[15,16]$. Generally, these are complex and mandate serial testing to rule out surface contamination with maternal fluids [15] or additional serological evidence of infection [16]. Broadly, intrauterine transmission can be reasonably confirmed, if the mother has been positive for SARS-CoV-2 within 14 days before or 2 days after birth and, the virus has been detected in amniotic fluid, placental tissue, neonatal blood or respiratory specimens collected within first 12 hours of birth, as well as in repeat neonatal blood or respiratory samples after 24 hours. If 
the amniotic fluid, placental tissue and early neonatal samples are negative but subsequent ones after 24 hours are positive, it is likely that the virus was acquired intrapartum or in early postpartum period.

Vivanti, et al. [17] made a strong case for transplacental transmission in a neonate who manifested neurological symptoms on day 3 of birth. The authors demonstrated the $\mathrm{E}$ and $\mathrm{S}$ genes of SARS-Co- $\mathrm{V}-2$ in the maternal blood and amniotic fluid along with high viral load in the placenta and histological evidence of placental inflammation. The nasopharyngeal and rectal swabs of the neonate collected 1 hour after birth, and then repeated on day 3 and 18 were positive for the two SARS-CoV-2 genes [19]. The neonate improved with symptomatic treatment and was discharged home. In another case of a symptomatic neonate whose nasopharyngeal swab was positive by RT-PCR at 24 and 48 hours after birth, SARSCoV-2 nucleocapsid protein and viral particles were demonstrated in the placental syncytiotrophoblast [18]. The neonate was separated at birth from its COVID-19 positive mother and subsequently recovered. Added to this conundrum is the demonstration of SARS-CoV-19 virus-specific antibodies (IgM and/or $\operatorname{IgG}$ ) in the neonatal serum despite negative nasopharyngeal swabs in a few cases [19]. While IgG antibodies can passively transfer across the placenta, the presence of IgM is intriguing. Whether the elevation and rapid decline of IgM antibody level noted in the above case represents fetal viremia that had subsequently cleared or signaled a falsepositive result due to cross-reactivity with other viruses is a matter of debate.

Breastmilk is unlikely to be a route of transmission. Among 48 milk samples from 32 infected women, only one tested positive for SARS-CoV-2 virus [20]. In two samples produced by a single woman, IgG but not IgM antibodies against SARS CoV 2 were detected. Chambers, et al. [21] showed that mere detection of viral RNA does not equate to infectivity, because the viral particles failed to replicate in tissue culture [21]. Recently, secretory antibodies against SARS-CoV-2 were demonstrated in a high proportion of human milk samples from 41 mothers with unknown COVID status during the pandemic [22]. Possibilities include an antibody response secondary to COVID-19 infection or the inherent characteristics of milk antibodies to have cross-reactive and poly-reactive properties against coronavirus and other related viruses. Thus, human milk might have a protective role against COVID-19 illness. In the AAP registry, the risk of COVID-19 infection among neonates isolated at birth $(22 / 1123 ; 2 \%)$ and those roomed-in $(21 /$ 974; 2.2\%) was similar [7]. The data from various national, population-based studies indicate that rooming- in and direct breastfeeding of infants born to mothers with confirmed or suspected SARS-CoV-2 infection do not increase the risk of infection if proper contact and droplet precautions are followed $[9,11]$.

\section{Clinical Manifestations in Infected Neonates}

Most neonates born to COVID-19 positive women are asymptomatic and carry only a small risk of acquiring the infection from mother $[11,23,24]$. However, they are at a higher risk of being born preterm $(30 \%)$ or by cesarean section ( $50 \%$ or greater) and may require intensive care for management of prematurity and other co-morbidities [6]. The incidence of symptoms in neonates varies as per proportion of preterm deliveries among different caseseries and reviews. Among 58 neonates with confirmed SARS-CoV-2 infection, $22 \%$ were asymptomatic, $41 \%$ presented with respiratory symptoms and $15 \%$ with fever [6]. Less common symptoms included poor feeding and lethargy (10\%) and gastrointestinal symptoms $(9 \%)$. The illness manifested beyond 24 hours of age, and most improved with symptomatic treatment. However, 38\% (22 of 58 positive neonates) required admission to neonatal unit and $17 \%$ required respiratory support. In the AAP registry, $30 \%$ of infected neonates $(n=43)$ manifested COVID-19 related symptoms. The duration of hospitalization was also longer in this group compared to COVID-19 negative neonates. Due to the overlap of usual morbidities of preterm and term neonates, it is difficult to tease out the contribution of SARS-CoV-2 infection to the reported symptoms and morbidities. Neonatal deaths due to COVID-related illnesses are uncommon $[8,11]$. However, follow-up has been reported only till hospital discharge and long-term outcomes are not known.

\section{Infectivity and Risk of Transmission of COVID-19}

Viral loads in children who are asymptomatic or have mild illness have been shown to be higher than hospitalized adults with severe disease [25]. Prolonged fecal excretion of SARS-CoV-2 has also been shown in children and could play a significant role in the transmission of COVID-19 disease [25,26]. Infected neonates may pose a higher risk to healthcare providers and family members, especially elderly who come in close contact with them or their excreta during caregiving activities. Face masks are not recommended for infants, and their care inherently requires close and repeated contacts.

\section{TESTING STRATEGY FOR COVID-19}

Web Table I provides a list of diagnostic modalities for COVID-19 and their application. The RT-PCR test to detect SARS Co-V-2 viral genome is the preferred diagnostic modality in all age groups, but the test should 
be interpreted along with clinical context. When the pretest probability of COVID-19 infection is high, a single negative RT-PCR test (sensitivity, 70\%; specificity, 99\%) does not help in ruling out an infection and the test needs to be repeated. Automated RT-PCR systems (CBNAAT or TrueNat) can be used where RTPCR testing is not available or quick turnaround is required e.g. emergency surgery. Rapid point of care antigen-based tests (RAT) on respiratory samples may have a role in triaging and rapid diagnosis. However, because of low sensitivity, if index of suspicion is high and test result is negative, confirmatory RT-PCR testing is recommended [27]. Due to fewer numbers of neonatal and pediatric cases, these recommendations are extrapolated from adult data [28].

\section{Optimal Testing Time in Neonates}

We examined the data extracted from published reports on neonates born to women with COVID-19 infection maintained by the Cochrane Gynecology and Fertility group [29]. Similar to the findings in the NNF survey, there were variations in the timing of testing. Therefore, the optimal testing time proposed in this article is derived from the knowledge of the viral infectivity and disease course in adults and children, and the testing recommendations by the National COVID-19 task force [27].

In neonates born to COVID-19 mothers, ideally a test should be done as early as possible after birth, within 12 hours (to find out vertical transmission, only for research purpose) and again after 5-10 days (as the initial test may

Table I Suggested Testing Strategy in Neonates Exposed to COVID-19 Infection

Rationale

1. Infected neonates requiring intensive or special care need to be isolated from other neonates

2. Health care workers and other caregivers need to take special precautions and wear appropriate personal protective equipment

3. Family members esp. elderly need to take special measures at home

4. Contributes to better understanding of neonatal SARS-CoV-2 epidemiology

\section{Choice of test}

- RT-PCR for SARS-CoV-2

- TruNat/CBNAAT

- Rapid antigen test (RAT) can be used as point of care( POC) test for triaging and rapid diagnosis. However, because of low sensitivity, a negative RAT needs to be confirmed with RT-PCR in symptomatic cases and if index of suspicion is high.

Serologic testing is not recommended to diagnose acute infection in neonates

Samples

- Combined naso-oropharyngeal swab or tracheal samples, if intubated

Interpretation of positive test

A positive result by RTPCR or TrueNat/CBNAAT or RAT is confirmatory

\begin{tabular}{|c|c|c|}
\hline Scenario & Timing of test & Repeat testing after initial negative test \\
\hline $\begin{array}{l}\text { Suspected perinatal transmission } \\
\text { Mother with COVID-19 infection detected } \\
\text { within } 14 \mathrm{~d} \text { before or within } 2 \mathrm{~d} \text { after delivery }\end{array}$ & $\begin{array}{l}\text { At birth or as soon as possible within } \\
12 \mathrm{~h} \text { of birth. Rooming-in should not } \\
\text { be postponed if testing is delayed. }\end{array}$ & $\begin{array}{l}\text { If the first test is negative, a repeat test is } \\
\text { recommended after } 5-10 \mathrm{~d} \text { of birth. Test } \\
\text { earlier if neonate becomes symptomatic. }\end{array}$ \\
\hline $\begin{array}{l}\text { History of exposure to COVID-19 positive } \\
\text { persons (including mother or family member } \\
\text { or healthcare provider) }\end{array}$ & $\begin{array}{l}\text { Asymptomatic high-risk contacts to be } \\
\text { tested once between day } 5 \text { and day } 10 \\
\text { of coming into contact. If symptomatic- } \\
\text { see below }\end{array}$ & $\begin{array}{l}\text { If symptoms develop following a negative } \\
\text { RAT test, a repeat testing by RT-PCR or } \\
\text { RAT should be done. }\end{array}$ \\
\hline
\end{tabular}

Symptomatic neonates (irrespective of history At the time of first evaluation of exposure) with onset at or beyond $48 \mathrm{~h}$ of life and presenting with acute respiratory (respiratory distress or apnea with or without cough, with or without fever) or sepsis like illness (fever, lethargy, poor feeding, seizures or diarrhea).

If negative, repeat the test in $24-48 \mathrm{~h}$ if the index of suspicion is high.

If positive, repeat the test only in severe illness. No re-testing is recommended prior to discharge from a COVID-19 facility after clinical recovery including transfer from a COVID facility to a non-COVID facility.

CBNAAT: Cartridge-based nucleic acid amplification test; COVID-19: Novel Corona virus disease; RT-PCR: Reverse transcriptase polymerase chain reaction; SARS-Co-V-2: Severe acute respiratory syndrome coronavirus 2; TruNat: Chip-based RT-PCR test. 
have false negatives and mother-infant dyad are generally roomed-in). However, for those neonates who are asymptomatic and otherwise fit to be discharged, the test can be scheduled as a pre-discharge sample at 24-72 hours of age (to avoid delay in discharge and missing sampling). Centers for Disease Control and Prevention, USA has also given similar guidelines [30]. The family should be advised to report to the nearest health facility for a repeat test if the neonate develops any symptoms or signs. In symptomatic neonates reporting to emergency, the test should be done at presentation. Based on the available evidence on neonatal SARS-CoV-2 transmission and the recommendations put forth by the National Task Force on COVID-19, we propose a testing strategy that is applicable for India in Table $\mathbf{I}$.

\section{CONCLUSION}

Although data on the incidence and outcomes of neonatal SARS-CoV-2 infection continue to emerge, there is much more to be learned. The evidence so far suggests that vertical transmission is uncommon and a greater proportion acquire infection postnatally through respi-ratory droplets or contact with infected mother or care-givers. Majority of neonates do not develop symptoms due to SARS-CoV-2 but the morbidities related to prematurity may necessitate intensive care and support. All neonates born to mothers with suspected or confirmed COVID-19 infection, regardless of presence of symp-toms should be tested. The awareness about neonatal COVID status promotes opportunities to implement infection prevention and control measures. Cases missed through lack of clinical suspicion or under-testing may facilitate the transmission of SARS-CoV-2 infection because asymptomatic infected neonates may serve as reservoirs of infection.

Contributors: All authors conceived the idea, reviewed the manuscript, analyzed and approved the manuscript.

Funding: None; Competing interests: None stated.

\section{REFERENCES}

1. World Health Organization. WHO Coronavirus Disease (COVID-19) Dashboard. Accessed 23 October 2020. Available from: https://covid19. who.int/?gclid $=$ EAIaIQob ChMItPnB-82x6wIVSK6WCh35jALrEAAYASABE gKUovD_BwE.

2. Hoang A, Chorath K, Moreira A, et al. COVID-19 in 7780 pediatric patients: A systematic review. E Clinical Medicine. 2020;24:100433.

3. Jiang L, Tang K, Levin M, et al. COVID-19 and multisystem inflammatory syndrome in children and adolescents. Lancet Infect Dis. 2020;20:E276-E88.

4. Gotzinger F, Santiago-Garcia B, Noguera-Julian A, et al. COVID-19 in children and adolescents in Europe: A multinational, multicentre cohort study. Lancet Child Adolesc Health. 2020;4:653-61.
5. Center for Disease Control and Prevention. Demographic Trends of COVID-19. Accessed on August 24, 2020. Available from: https://www.cdc.gov/covid-data-tracker/ index.html\#demographics

6. Dhir SK, Kumar J, Meena J, Kumar P. Clinical features and outcome of SARS-CoV-2 infection in neonates: A systematic review. J Trop Pediatr. 2020?:fmaa059 [Published online August 28, 2020].

7. Chawla D, Chirla D, Dalwai S, et al. Perinatal-Neonatal Management of COVID-19 Infection - Guidelines of the Federation of Obstetric and Gynaecological Societies of India (FOGSI), National Neonatology Forum of India (NNF), and Indian Academy of Pediatrics (IAP). Indian Pediatr. 2020;57:536-48.

8. American Academy of Pedaitrics (AAP), Section on Neonatal Perinatal Medicine. National registry for surveillance and epidemiology of perinatal COVID-19 infection. Accessed 5 Sep, 2020. Available from: https:// twitter.com/AAPneonatal/status/1301301988454535168/ photo/1

9. Nayak AH, Kapote DS, Fonseca M, et al. Impact of the coronavirus infection in pregnancy: A preliminary study of 141 patients. J Obstet Gynaecol India. 2020;70:256-61.

10. Ayed A, Embaireeg A, Benawadth A, et al. Maternal and perinatal characteristics and outcomes of pregnancies complicated with COVID-19 in Kuwait. medRxiv 2020.07.10.20150623v1 [preprint].

11. Knight M, Bunch K, Vousden N, et al. Characteristics and outcomes of pregnant women admitted to hospital with confirmed SARS-CoV-2 infection in UK: National population based cohort study. BMJ. 2020;369:m2107.

12. Maraschini A, Corsi E, Salvatore MA, Donati S. Coronavirus and birth in Italy: results of a national populationbased cohort study. medRxiv. 2020.2006.2011.20128652 [preprint].

13. Oncel MY, Akin IM, Kanburoglu MK, et al. A multicenter study on epidemiological and clinical characteristics of 125 newborns born to women infected with COVID-19 by Turkish Neonatal Society. [published online ahead of print, 2020 Aug 10] [published correction appears in Eur J Pediatr. 2020 Aug 22]. Eur J Pediatr. 2020;1-10.

14. Vivanti AJ, Mattern J, Vauloup-Fellous C, et al. Retrospective description of pregnant women infected with severe acute respiratory syndrome coronavirus 2, France. Emerg Infect Dis. 2020;26:2069-76.

15. Shah PS, Diambomba Y, Acharya G, Morris SK, Bitnun A. Classification system and case definition for SARS-CoV-2 infection in pregnant women, fetuses, and neonates. Acta Obstet Gynecol Scand. 2020;99:565-8.

16. Blumberg DA, Underwood MA, Hedriana HL, Lakshminrusimha S. Vertical Transmission of SARS-CoV2: What is the Optimal Definition? Am J Perinatol. 2020;37:769-772.

17. Vivanti AJ, Vauloup-Fellous C, Prevot S, et al. Transplacental transmission of SARS-CoV-2 infection. Nat Commun. 2020;11:3572.

18. Sisman J, Jaleel MA, Moreno W, et al. Intrauterine transmission of SARS-COV-2 infection in a preterm infant. Pediatr Infect Dis J. 2020;39:e265-e 7. 
19. Zeng H, Xu C, Fan J, et al. Antibodies in Infants Born to Mothers With COVID-19 Pneumonia. JAMA. 2020. 323: 1848-9.

20. Lackey KA, Pace RM, Williams JE, et al. SARS-CoV-2 and human milk: What is the evidence? Matern Child Nutr. 2020:e13032.

21. Chambers C, Krogstad P, Bertrand K, et al. Evaluation for SARS-CoV-2 in breast milk from 18 infected women. JAMA. 2020;324:1347-8

22. Demers-Mathieu V, Dung M, Mathijssen GB, et al. Difference in levels of SARS-CoV-2 S1 and S2 subunitsand nucleocapsid protein-reactive $\mathrm{SIgM} / \operatorname{IgM}$, IgG and SIgA/IgA antibodies in human milk. J Perinatol. 2020:1-10 [Published 2020 Sep 1].

23. Ludvigsson JF. Systematic review of COVID-19 in children shows milder cases and a better prognosis than adults. Acta Paediatr. 2020;109:1088-95.

24. Liguoro I, Pilotto C, Bonanni M, et al. SARS-COV-2 infection in children and newborns: A systematic review. Eur J Pediatr. 2020;179:1029-46.

25. Xing $\mathrm{YH}, \mathrm{Ni} \mathrm{W}, \mathrm{Wu} \mathrm{Q}$, et al. Prolonged viral shedding in feces of pediatric patients with coronavirus disease. J Microbiol Immunol Infect. 2019;53:473-80.

26. Li X, Xu W, Dozier M, et al. The role of children in transmission of SARS-CoV-2: A rapid review. J Glob Health.2020;10:011101.

27. Indian Council of Medical Research (ICMR).Advisory on
Strategy for COVID-19 Testing in India (Version VI, dated 4th September 2020). Accessed September 5, 2020. Available from: https://www.icmr.gov.in/pdf/covid/ strategy/Testing_Strategy_v6_04092020.pdf

28. Dinnes J, Deeks JJ, Adriano A, et al. Rapid, point-of-care antigen and molecular-based tests for diagnosis of SARSCoV-2 infection. Cochrane Database Syst Rev. 2020;8: CD013705.

29. Cochrane Gyanecology and Fertility group. Excel sheet Perinatal outcomes in COVID-19 infection. Accessed 1 Sept, 2020. Available from: https://cgf.cochrane.org/news/ covid-19-coronavirus-disease-fertility-and-pregnancy

30. Centers for Disease Control and Prevention. Evaluation and Management Considerations for Neonates at risk for COVID-19. Accessed September 04, 2020. Available from: https://www.cdc.gov/coronavirus/2019-ncov/hcp/ caring-for-newborns.html

31. Watson J, Whiting PF, Brush JE. Interpreting a covid-19 test result. BMJ. 2020;369:m1808.

32. Ridgway JP, Pisano J, Landon E, Beavis KG, Robicsek A. Clinical Sensitivity of Severe Acute Respiratory Syndrome Coronavirus 2 Nucleic Acid Amplification Tests for Diagnosing Coronavirus Disease 2019. Open Forum Infect Dis.2020;7:ofaa315 [Published 2020 Jul 24].

33. Tang MS, Hock KG, Logsdon NM, et al. Clinical performance of two SARS-CoV-2 serologic assays. Clin Chem. 2020;66:1055-62. 